\title{
DETERMINATION OF RESONANCE TERMS \\ USING OPTICAL OBSERVATIONS \\ OF TWO METEOSAT SATELLITES
}

\author{
U. HUGENTOBLER, T. SCHILDKNECHT AND G. BEUTLER \\ Astronomical Institute, University of Berne \\ Berne, Switzerland
}

\begin{abstract}
During an observation campaign in winter 94/95 astrometric positions from Meteosat 4 and 5 were acquired at the Zimmerwald observatory using a CCD camera mounted in the prime focus of the $0.5 \mathrm{~m}$ Satellite Laser Ranging telescope. The measurements cover a time interval of four months, their precision is of the order of $0^{\prime \prime} 7$.

The modeling of radiation pressure for the small, cylindrically shaped satellites is relatively easy and they are therefore excellent objects to probe the geopotential. The orbital parameters and the radiation pressure coefficients for the two satellites as well as the resonant coefficients $C_{22}, S_{22}$ of the geopotential were determined by a single least square adjustment procedure including all the Zimmerwald observations. The relative errors estimated for the terms $C_{22}$ and $S_{22}$ are of the order of $1 \div 3 \cdot 10^{-4}$.
\end{abstract}

\section{Introduction}

It is well known since the beginning of the space age that the ellipticity of the Earth's equator causes a resonant longitude drift for satellites in a geosynchronous orbit [e.g. (Sehnal, 1960), (Kovalevsky, 1961), (Blitzer, 1962)]. The dominant terms responsible for this drift are the coefficients $C_{22}$ and $S_{22}$ in the harmonic series expansion of the geopotential. The libration period of a satellite in resonance is of the order of 1000 to 2000 days while the semimajor axis may vary by up to $\pm 35 \mathrm{~km}$.

Because geostationary satellites are especially sensitive to the geopotential terms $C_{22}$ and $S_{22}$, observations of their librating motion can be used to determine these coefficients. The first determination of the two geopotential

I. M. Wytrzyszczak, J. H. Lieske and R. A. Feldman (eds.), Dynamics and Astrometry of Natural and Artificial Celestial Bodies, 355, 1997.

(C) 1997 Kluwer Academic Publishers. Printed in the Netherlands. 
coefficients using observations of geostationary satellites was performed by (Wagner, 1965) who processed radio-tracking data from SYNCOM 2. (Catalano et al., 1983) proposed the determination of the resonant coefficients and their temporal variation using optical observations of geostationary satellites.

In $1987 \mathrm{~A}$. M. Nobili established the research project COGEOS (International Campaign for Optical Observations of Geosynchronous Satellites). The aim of COGEOS is the detection of possible time variations of the geopotential coefficients which are resonant with the orbits of geosynchronous satellites. The observations presented in this paper were acquired in the context of this program.

\section{Instrumentation, Measurement Technique, Observations}

The observations were obtained at the $0.5 \mathrm{~m}$ Satellite Laser Ranging (SLR) telescope in Zimmerwald, equipped with a thermoelectrically cooled CCD camera in its prime focus. The $512 \times 512$ pixel Photometrics chip with a pixel size of $20 \mu \mathrm{m} \times 20 \mu \mathrm{m}$ gives a field of view of $35^{\prime}$ and a mapping scale of $4.16^{\prime \prime} /$ pixel. Using the time subsystem of the SLR station and a special shuttering technique (Schildknecht, 1994) the centroids of the satellite image could be tagged to within $1 \mathrm{~ms}$ with respect to UTC.

The $0.5 \mathrm{~m}$ Zimmerwald SLR telescope was dismounted in May 1995 and a new $1 \mathrm{~m}$ telescope was installed. After first light in May 1996 the new instrument, which is designed for both, Satellite Laser Ranging and astrometric observations, currently (July 1996) undergoes its final optical adjustments and will be operational by September 1996 .
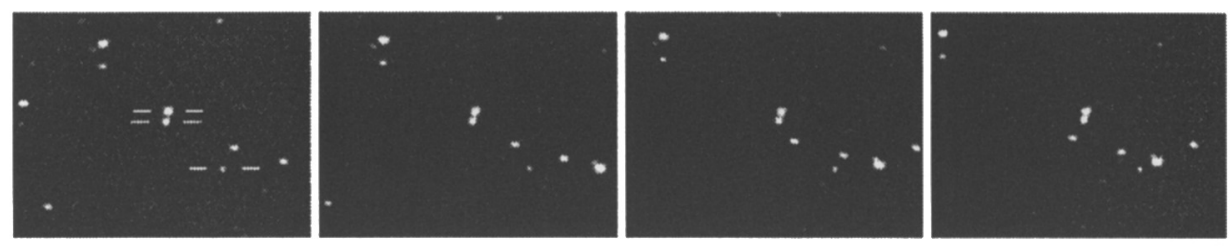

Figure 1. Meteosat 5 (bottom right) together with two other geostationary satellites in front of the moving stellar background. This series of frames was taken in Zimmerwald on January 31, 1995, with fixed telescope and at 8 second intervals. Integration time was 1 second. The field covers $14^{\prime} \times 10^{\prime}$.

Due to the small field of view a special technique is used for measuring astrometric satellite positions (Schildknecht, 1993): Geostationary satellites are observed with fixed telescope (see Figure 1). A series of short exposures ( 1 second) taken during a close passage of a reference star allows the calibration of the scale and the camera orientation, and the measurement of 
the satellite positions. Through this procedure we acquire about 10 astrometric positions relative to a single reference star for each series of close encounter. Additional off-line calibration sessions are necessary to monitor the distortions in the field of view.

The exposures are processed in real time in order to avoid storage on disk. This is mandatory because of the large amount of data represented by the CCD frames. The centroids of the satellite and reference star images are determined by a center of light algorithm which provides an accuracy of about 0.1 pixels for faint images.

The astrometric accuracy for geostationary satellites obtained with the SLR telescope is between 0.3 and 0.7 depending on the brightness of the satellite. For the new Zimmerwald telescope an accuracy of $0 \prime 1$ is expected.

We used the PPM (Positions and Proper Motions) and CMC (Carlsberg Meridian Circle) astrometric catalogues for the positions of the reference stars.

The observation campaign for Meteosat 4 and 5 was timed in such a way that neither of the two satellites crossed the Earth's or the Moon's shadow during the observation period. This avoids problems with the modeling of the radiation pressure force. A total of 1300 observations in 157 single encounter series was acquired during 20 nights in winter 94/95. Table 1 summarizes the data for the two satellites. The precision of single observations for the relatively faint satellites is between 0.5 and 0.7 .

TABLE 1. Observations of Meteosat 4 and 5.

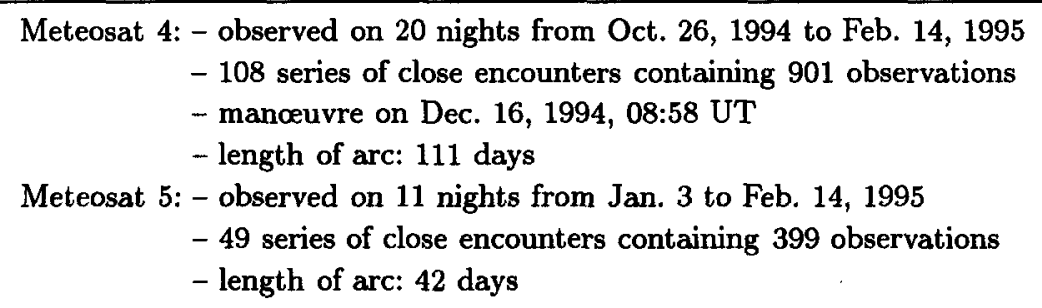

\section{Determination and Combination of Orbits}

The observations of the two satellites were split into three arcs with lengths of 42 to 45 days. For each arc we generated an orbit using the ORBDET program, developed at the Astronomical Institute of the University of Berne. The force model includes:

- Earth potential model JGM-3 to degree and order 10,

- gravity of Sun and Moon using JPL DE200 ephemerides,

- direct solar radiation pressure, 
- tidal potential due to Sun and Moon,

- precession and nutation according to the IAU 1976 and 1980 resolutions,

- UT1-UTC correction and polar motion from IERS.

The orbit integration was performed in the inertial system J2000. The program allows the estimation of Keplerian or nonsingular orbital elements, radiation pressure coefficients, and gravity potential coefficients.

In order to use all observations within one parameter estimation process we combined the three arcs on the normal equation level by modelling the manœuvre of Meteosat 4 with a velocity change at the manœuvre epoch and by requiring the dynamical parameters (potential terms) for the three arcs to be equal. This procedure is described by (Beutler et al., 1996). The orbit determination and the orbit combination steps were iterated until the termination criterion was met.

The combined solution contains a total of 19 estimated parameters: $2 \times 6$ initial conditions for Meteosat 4 and 5,2 $\times 1$ direct solar radiation pressure coefficients for Meteosat 4 and 5, 3 manœuvre parameters (velocity changes) for Meteosat 4, and 2 geopotential terms $C_{22}$ and $S_{22}$.

The residuals of the combined orbit determination are given in Figure 2. They show no systematic behaviour, the observations of the two satellites clearly are well represented by the model described above. The rms for the single observations is of the order of 0.65 .

Table 2 lists the estimated manœuvre parameters for Meteosat 4 . The associated formal errors are rather small. Obviously the satellite performed an inclination manœuvre in order to maintain the orbital plane at its nominal inclination.

A simple solar radiation pressure model which considers only the direct effect is quite satisfactory. The more sophisticated model proposed by (Veillet, 1990) taking into account the cylindrical shape of the satellites, does not significantly improve the results.

TABLE 2. Manœuvre Parameters for Meteosat 4.

\begin{tabular}{llccc}
\hline Velocity change & radial & $(-32.7 \pm 6.1) \cdot 10^{-4}$ & $\mathrm{~m} / \mathrm{s}$ \\
& alongtrack & $(-1.09 \pm 0.16) \cdot 10^{-4}$ & $\mathrm{~m} / \mathrm{s}$ \\
& crosstrack & $-0.2103 \pm$ & 0.0009 & $\mathrm{~m} / \mathrm{s}$ \\
\hline
\end{tabular}

\section{The Resonant Geopotential Coefficients}

The calculated values for the resonant geopotential terms are listed in Table 3 together with the corresponding values from the gravity model JGM-3. 

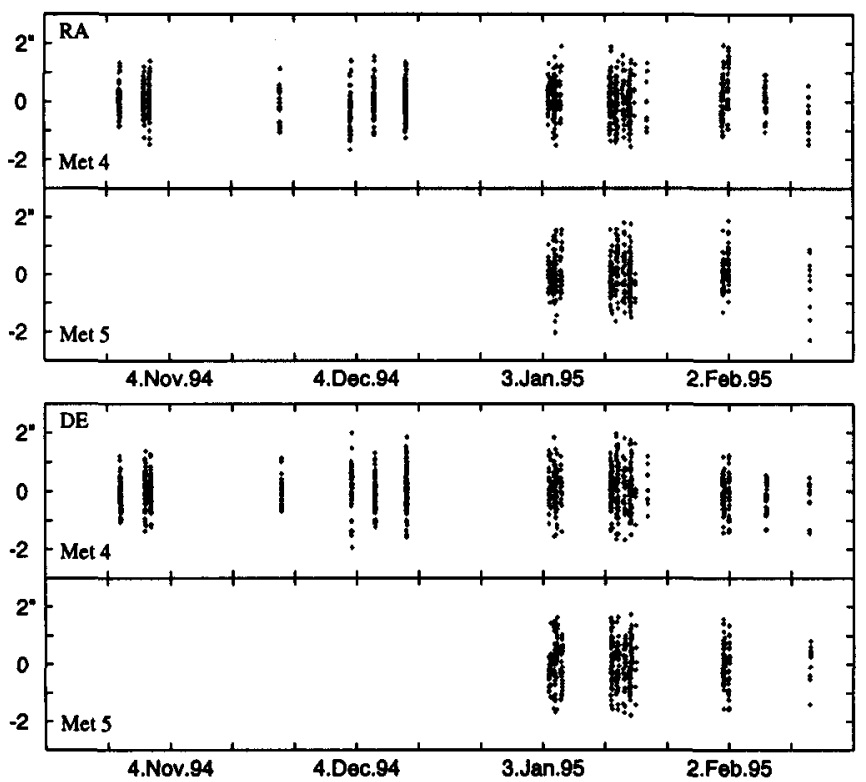

Figure 2. Residuals in right ascension (top) and declination (bottom) for Meteosat 4 and 5 from the combined orbit determination.

The relative precision of the terms is

$$
\frac{\sigma\left(C_{22}\right)}{C_{22}}=2.8 \cdot 10^{-4} \quad \text { and } \quad \frac{\sigma\left(S_{22}\right)}{S_{22}}=1.1 \cdot 10^{-4}
$$

which is about an order of magnitude larger than the relative formal errors of $1.5 \cdot 10^{-5}$ and $2.6 \cdot 10^{-5}$ for $C_{22}$ and $S_{22}$ respectively in the JGM-3 model. It has to be kept in mind, however, that the results presented in this paper were produced with a relatively small effort, the observations being acquired during a short campaign with a telescope of modest optical quality.

TABLE 3. Resonant Geopotential Coefficients.

\begin{tabular}{lrr}
\hline Terms & Combined Solution & \multicolumn{1}{c}{ JGM-3 } \\
\hline$C_{22}$ & $(2.43825 \pm 0.00069) \cdot 10^{-6}$ & $2.43926 \cdot 10^{-6}$ \\
$S_{22}$ & $(-1.40056 \pm 0.00016) \cdot 10^{-6}$ & $-1.40027 \cdot 10^{-6}$ \\
\hline
\end{tabular}

Simulations based on a similar configuration of the observations as in the COGEOS campaign and adopting an astrometric accuracy of 0 " 1 show that 
the formal errors of the resonant geopotential coefficients are improved by a factor of ten. In other words, astrometric methods are competitive with other methods for the determination of the geopotential terms $C_{22}$ and $S_{22}$, provided one uses a good telescope and high precision astrometric catalogues (such as Hipparcos).

\section{Conclusions}

Optical astrometric observations of geostationary satellites proved to be useful for the determination of the resonant geopotential terms $C_{22}$ and $S_{22}$. An astrometric accuracy of $0 \prime \prime 1$ as expected for the Zimmerwald $1 \mathrm{~m}$ telescope makes the precision of the coefficients competitive with the best geopotential models available today. With short campaigns and with a limited effort for both, observations and processing, the resonant low order coefficients can be monitored in order to detect temporal variations or to establish upper limits for these variations. The combination of astrometric observations of several satellites at different longitudes and from different observatories will further strengthen the determination of the resonant geopotential coefficients.

Acknowledgements. We thank A.M. Nobili, P. Paquet, F. Barlier, and G. Appleby for initiating and coordinating the COGEOS observation campaign, G. Ferrand for providing information about the Meteosat satellites. The work was partly founded by the Swiss National Science Foundation.

\section{References}

Beutler, G., Brockmann, E., Hugentobler, U., Mervart, M., Rothacher, M., and Weber, R.: 1996, "Combining consecutive short arcs into long arcs for precise and efficient GPS orbit determination", J. Geodyn. 70, 287-299.

Blitzer, L., Boughton, E.M., Kang, G., and Page, R.M.: 1962, "Effect of ellipticity of the equator on 24-hour nearly circular satellite orbits", J. Geophys. Res. 67, 329-335.

Catalano, S., McCrosky, R., Milani, A., and Nobili, A.M.: 1983, "Optical tracking of synchronous Earth's satellites for geophysical purposes", J. Geophys. Res. 88, 669676.

Kovalevsky, J.: 1961, First Int. Symposium on Analytical Astrodynamics, UCLA.

Schildknecht, T., Hugentobler, U., and Verdun, A.: 1993, "CCD astrometry of artificial satellites", in: Dynamics and Astrometry of Natural and Artificial Celestial Bodies (K. Kurzyńska, F. Barlier, P.K. Seidelmann, I. Wytrzyszczak, eds), Astronomical Observatory, Poznań, 103-108.

Schildknecht, T.: 1994, "Optical astrometry of fast moving objects using CCD detectors", Ph.D. thesis, Geodätisch-geophysikalische Arbeiten in der Schweiz, Vol. 49.

Sehnal, L.: 1960, "The perturbations of the orbit of the stationary satellite of the Earth", Bull. Astron. Inst. Czech. 11, 132-135.

Veillet, Ch., Metris, G., and Boudon, Y.: 1990, "Status report of Cerga/OCA", Third COGEOS Workshop, Brussels, June 11-12, 1990.

Wagner, C.A.: 1965, "A determination of Earth equatorial ellipticity from seven months of Syncom 2 longitude drift", J. Geophys. Res. 70, 1566-1568. 